\title{
Near-Capacity Cooperative Space-Time Coding Employing Irregular Design and Successive Relaying
}

\author{
Lingkun Kong, Soon Xin Ng, Robert G. Maunder and Lajos Hanzo
}

\begin{abstract}
In this paper, we develop a capacity-approaching Cooperative Space-Time Coding (CSTC) scheme employing irregular design for a twin-relay aided network as an extension of our previous work cast in the context of a half-duplex singlerelay-aided network. For the sake of recovering the multiplexing loss imposed by a half-duplex three-terminal network, we employ a successive relaying protocol in this paper, where an additional relay node is activated. Hence, in order to design a near-capacity coding system, first the capacity and the achievable informationrate of a specific space-time coding aided scheme are quantified for the successive relaying aided channel. More specifically, the cooperative space-time codes employed at the source and the relays are jointly designed with the aid of EXtrinsic Information Transfer (EXIT) charts for the sake of high-integrity operation at Signal-to-Noise Ratios (SNRs) close to the corresponding successive relaying channel's capacity. Furthermore, unlike in the halfduplex single-relay based system, the destination node performs frame-by-frame Successive Interference Cancellation (SIC) aided iterative detection, in order to mitigate the efforts of multipleaccess interference. Finally, our numerical results demonstrate that our proposed Irregular Cooperative Space-Time Coding (IrCSTC) scheme is capable of near-capacity operation in the successive relaying aided network, which is an explicit benefit of our joint source-and-relay transceiver design.
\end{abstract}

Index Terms-Irregular cooperative space-time code, iterative detection, irregular convolutional code, EXIT charts.

\section{INTRODUCTION}

\section{A. Background}

C OOPERATIVE communications [1], [2] have drawn substantial research attention in the past few years, which combine the benefits of distributed Multiple-Input MultipleOutput (MIMO) systems with relay-aided techniques. Relaying techniques may be employed in diverse communication scenarios, such as cellular networks, wireless ad-hoc and sensor networks, and wireless local area networks, in order to improve the attainable transmission efficiency and/or reliability. Specifically, in a relay aided network where the nodes (users) are equipped with either single or multiple antennas, cooperative communications allow the nodes (users) to assist each other in forwarding (relaying) all messages to the destination, rather than transmitting only their own messages. Two main aspects of relay-aided networks have been investigated in the literatures, namely the fundamental limits of transmissions over relay channels [3]-[9], and the development of practical cooperative protocols and transmission schemes [2], [10], [11].

School of Electronics and Computer Science, University of Southampton, SO17 1BJ, United Kingdom. Email: \{1k06r,sxn,rm,lh\}@ecs.soton.ac.uk.

The financial support of the China-UK Scholarship Council, as well as that of the EPSRC UK, and of the EU under the auspices of the Optimix project is gratefully acknowledged.
The information theoretic study of the relay aided channel's capacity was pioneered by Cover and El Gamal [3]. Specifically, they derived the exact capacity expression under certain conditions and evaluated both the lower and upper bounds of the achievable modem-mode-related information rates. Recent information theoretic studies considered the Gaussian relay channel in the context of multiple relay nodes [8], and relay nodes operating in either full- or half-duplex mode [4], as well as multi-antenna aided relaying systems [12] and twoway relay channels [9].

In addition to the above-mentioned information theoretic investigations, there are numerous studies on practical transmission schemes designed for relaying systems. For the sake of improving the achievable diversity gain of practical relay-aided half-duplex networks, numerous cooperative protocols [1], [2], [10], [13] have been proposed. However, in most threeterminal aided cooperative scenarios a significant multiplexing loss is incurred compared to classic direct transmissions due to the half-duplex constraint of practical transceivers. For the sake of recovering the multiplexing loss as well as maintaining the relays' low complexity, a successive relaying protocol was proposed in [14], which incorporated an additional relay in the network, where the two relays transmit in turn. However, as mentioned in [14], a sophisticated channel code was required for achieving near-error-free detection at the relay nodes and hence to prevent error-propagation, which was beyond the scope of [14].

On the other hand, inspired by the classic turbo codes used in non-cooperative communication scenarios, Distributed Turbo Codes (DTC) [15] have been proposed for "distributed MIMO" systems, which benefit from a turbo processing gain, and therefore they are particularly suitable for operation at low SNRs. However, DTCs suffer from having an imperfect communication link between the component decoders constituted by the source and relay nodes. In order to circumvent this drawback, a three-component Distributed Turbo Trellis Coded Modulation (DTTCM) scheme has been proposed in [16], which takes into consideration the realistic condition of having an imperfect source-to-relay communication link. The DTTCM scheme of [16] was designed using EXtrinsic Information Transfer (EXIT) chart analysis [17], [18], and it was capable of minimizing the decoding error probability at the relay. As a benefit, it performed close to its idealized counterpart that assumes perfect decoding (no decoding errors) at the relay. However, the DTTCM of [16] still fails to approach the corresponding relay channel's capacity. On the other hand, several capacity-approaching turbo coding schemes were proposed in [19] and [20] by Zhang and Duman for full-duplex and half-duplex relay systems, respectively, 
although the authors did not aim for finding the optimal coding schemes under different relay network configurations ${ }^{1}$.

\section{B. Contribution}

In [22], an Irregular Distributed Space-Time Coding (IrDSTC) scheme was proposed for the sake of approaching the three-terminal half-duplex relay system's capacity. Due to the half-duplex constraint ${ }^{2}$, the relay system's effective throughput in [22] was reduced by a factor of two. In this paper, we propose a more general Irregular Cooperative Space-Time Coding (Ir-CSTC) scheme for a twin-relay aided network, where the successive relaying protocol is employed for the sake of recovering the half-duplex relaying loss. In order to design a capacity-approaching cooperative coding scheme for the successive relaying scenario, we first derive both the upper and lower bounds of the Continuous-input Continuousoutput Memoryless Channel's (CCMC) [23], [24] capacity as well as the bounds of the information rates constrained by independent and uniformly distributed (i.u.d) discrete inputs for the successive relaying aided uncorrelated Rayleigh fading channel. Furthermore, a joint source-and-relay mode design procedure is proposed for the twin-relay aided network. Specifically, the distributed code components used at the source and relays are optimally designed. As a benefit, the IrCSTC scheme becomes capable of approaching the Discreteinput Continuous-output Memoryless Channel's (DCMC) [23], [24] capacity (constrained information rate) in the context of the successive relaying aided network, when the frame length is sufficiently long (e.g. $10^{5}$ bits), while approaching the transmission efficiency of classic direct transmission operating without relaying. Most importantly, it will be demonstrated that our joint source-and-relay mode design procedure is capable of finding the optimal cooperative coding scheme under arbitrary relay network configuration.

The rest of this paper is organised as follows. The system model and the successive relaying protocol are described in Section II. Section III specifies the encoding and decoding processes of the Ir-CSTC scheme designed for the successive relaying aided network. The CCMC capacity and the upper as well as lower bounds of the successive relaying channel's DCMC capacity (constrained information rate) and the EXIT chart aided joint source-and-relay mode design are detailed in Section IV, while our simulation results and discussions are provided in Section V. Finally, we conclude in Section VI.

\section{System Model AND PROTOCOL DESCRIPTION}

\section{A. System Model}

We consider the four-terminal successive relaying aided network of Fig. 1, where a single source $s$ is equipped with $N_{s}$

${ }^{1}$ Some other low-complexity distributed space-time codes can be found in [21].

${ }^{2}$ The reason of assuming half-duplex terminals relies on the fact that current limitations in radio transceiver implementations preclude the terminals from full-duplex operation, i.e. from transmitting and receiving at the same time in the same frequency band. To elaborate a little further, indeed, it would be beneficial in capacity terms to transmit and receive at the same time, but this would result in the following problems. The received signal may be almost $100 \mathrm{~dB}$ lower than the transmit signal. Hence the smallest leakage of the transmitted signal would desensitize the receiver's Automatic Gain Control (AGC), hence potentially drowning the received signal.

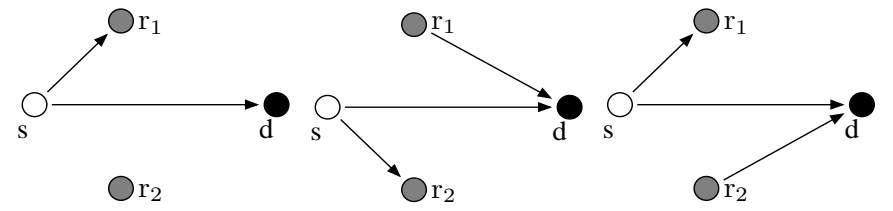
(a) Phase 1
(b) Phase 2
(c) Phase 3

S \begin{tabular}{|l|l|l|l|l|}
\hline 1 & 2 & 3 & 4 & 5 \\
\hline
\end{tabular}

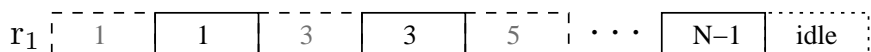

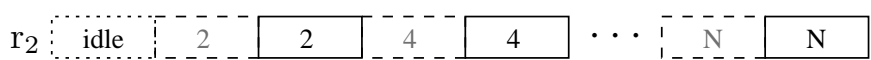

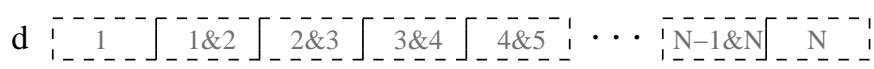

(d) Frame structure, solid box for transmitted signal, dashed box for received signal and dotted box for idle status

Fig. 1. System model for the successive relaying aided network, where the interference between the relays is negligible.

antennas, and intends to communicate with the destination $d$ having $N_{d}$ antennas. The two relays $r_{1}$ and $r_{2}$ are equipped with $N_{r_{1}}$ and $N_{r_{2}}$ antennas, respectively, and each relay $r_{i}$ can be either a mobile user or a fixed relay. Compared to the conventional single-relay-aided scheme, it is clearly seen that one additional relay is required to support the successive relaying, which potentially increases the overall infrastructure cost. However, this can be avoided, when the relays are constituted by inactive mobile users. To obey the realistic limitations of practical transceivers, all nodes in the network obey the half-duplex constraint, i.e. a node cannot transmit and receive simultaneously. Furthermore, we consider a similar scenario to that of [25], where the relays were said to have "weak interconnections". In our case, the interference between the relays is considered to be negligible compared to the desired signal power, especially when no line-of-sight (LOS) transmission exists among the relays. As in [26], we model the communication links between the nodes of Fig. 1 as being subjected to both free-space path loss as well as to uncorrelated Rayleigh fading, except for the link between the relays. Hence, as a benefit of the commensurately reduced distance and path loss, we achieve a proportional geometrical-gain [26] for the source-to-relay (SR) links and the relay-to-destination (RD) links with respect to the source-to-destination (SD) link, which are denoted by $G_{s r_{1}}, G_{s r_{2}}$ and $G_{r_{1} d}, G_{r_{2} d}$, respectively. Naturally, the geometrical-gain of the SD link with respect to itself is unity, i.e. $G_{s d}=1$. In this paper, we assume that the relays are closer to the source than to the destination ${ }^{3}$, while both the source and relays are far away from the destination, namely we have $G_{s r_{i}}>G_{r_{i} d}, i=1,2$. In this scenario, the relays benefit from a higher received signal power than the destination, which facilitates the employment of near-perfect Decode-and-Forward (DF) relaying.

${ }^{3}$ In [27] and [28], the effects of relay position were investigated and the authors considered the benefits of power allocation as a countermeasure. 


\section{B. Protocol Description}

We split the source transmissions into different identicallength frames. As illustrated in Fig. 1, the transmission scheduling of the successive relaying protocol can be described as follows ${ }^{4}$. In Phase 1 of Fig. 1(a), $s$ transmits frame $1 ; r_{1}$ listens to $s ; r_{2}$ remains silent and $d$ receives frame 1 from $s$. In Phase 2 of Fig. 1(b), $s$ transmits frame $2 ; r_{1}$ decodes, reencodes and forwards frame $1 ; r_{2}$ listens to $s$ and $d$ receives frame 1 from $r_{1}$ and frame 2 from $s$. By contrast, during Phase 3 seen in Fig. 1(c), $s$ transmits frame 3; $r_{2}$ decodes, re-encodes and forwards frame $2 ; r_{1}$ listens to $s$ and $d$ receives frame 2 from $r_{2}$ and frame 3 from $s$. This progress is repeated in this manner until Phase $N$. In Phase $(N+1), s$ and $r_{1}$ (or $r_{2}$ ) keep silent. Then $r_{2}$ (or $r_{1}$ ) decodes, re-encodes and forwards frame $N$, while $d$ receives frame $N$ from $r_{2}$ (or $r_{1}$ ). The frame structure of the successive relaying scheme is further illustrated in Fig. 1(d). It is clearly seen that in our successive relaying scheme, $(N+1)$ communication phases are required to convey $N$ frames of information with the aid of two relays. Hence, the efficiency of classic direct transmission is approached, provided that $N$ is sufficiently high. The vector hosting the received signal at the relay $r_{i}$ can be formulated as:

$$
\mathbf{y}_{r_{i}}=\sqrt{G_{s r_{i}}} \mathbf{H}_{s r_{i}} \mathbf{c}_{s}+\mathbf{n}_{r_{i}} .
$$

By contrast, the signal vector received at the destination $d$ during the first and last transmission phase can be expressed as:

$$
\mathbf{y}_{d}=\sqrt{G_{s d}} \mathbf{H}_{s d} \mathbf{c}_{s}+\mathbf{n}_{d}
$$

and

$$
\mathbf{y}_{d}=\sqrt{G_{r_{i} d}} \mathbf{H}_{r_{i} d} \mathbf{c}_{r_{i}}+\mathbf{n}_{d}, \quad i=1 \text { or } 2,
$$

respectively, while the signal received by $d$ from $s$ during the intermediate phase is contaminated by the interfering signals received from the relay, which is formulated as:

$$
\mathbf{y}_{d}=\sqrt{G_{s d}} \mathbf{H}_{s d} \mathbf{c}_{s}+\sqrt{G_{r_{i} d}} \mathbf{H}_{r_{i} d} \mathbf{c}_{r_{i}}+\mathbf{n}_{d}, \quad i=1 \text { or } 2,
$$

where $\mathbf{y}_{r_{i}}=\left[y_{r_{i}, 1}, \ldots, y_{r_{i}, N_{r_{i}}}\right]^{T}$ is the $N_{r_{i}}$-element vector of the signals received at relay $r_{i}$. Furthermore, $\mathbf{y}_{d}=\left[y_{d, 1}, \ldots, y_{d, N_{d}}\right]^{T}$ is the $N_{d}$-element vector of the signals received at the destination, while $\mathbf{H}_{s r_{i}} \in \mathbb{C}^{N_{r_{i}} \times N_{s}}, \mathbf{H}_{s d} \in \mathbb{C}^{N_{d} \times N_{s}}$ and $\mathbf{H}_{r_{i} d} \in \mathbb{C}^{N_{d} \times N_{r_{i}}}$ are the corresponding channel matrices having independent and identically complex Gaussian distributed elements with a zero mean and a variance of 0.5 per dimension. Furthermore, $\mathbf{c}_{s}=\left[c_{s, 1}, \ldots, c_{s, N_{s}}\right]^{T}$ and $\mathbf{c}_{r_{i}}=\left[c_{r_{i}, 1}, \ldots, c_{r_{i}, N_{r_{i}}}\right]^{T}$ are the $N_{s^{-}}$ element vector of the signals transmitted from the source $s$ and the $N_{r_{i}}$-element vector of signals transmitted from the relay $r_{i}$, respectively. In this paper, we assume that the source and relays transmit at the same power $P_{0}$. Finally, $\mathbf{n}_{r_{i}}=\left[n_{r_{i}, 1}, \ldots, n_{r_{i}, N_{r_{i}}}\right]^{T}$ and $\mathbf{n}_{d}=\left[n_{d, 1}, \ldots, n_{d, N_{d}}\right]^{T}$ are the corresponding $N_{r_{i}}$-element and $N_{d}$-element AWGN vectors, both with each element having a zero mean and a variance of $N_{0} / 2$ per dimension.

\footnotetext{
${ }^{4}$ In this contribution, we only consider the scenario, where the two relays are always active in assisting the source. The cooperative systems, where the relays are not always cooperating are investigated in [13] and [21].
}

\section{IrRegular CoOperative Space-Time Coding FOR SUCCESSIVE RELAYING NETWORK}

In [22], we proposed a novel Ir-DSTC coding scheme for the three-terminal relay-aided network. In this paper, we will propose a general Cooperative Space-Time Coding (CSTC) scheme using irregular design for the four-terminal successive relaying aided network, which will be shown in Section IV to be capable of approaching the DCMC capacity limit for the successive relaying channels.

\section{A. Distributed Encoding at the Source and Relays}

As seen in Fig. 2, at the source of the four-terminal successive relaying aided network, we use a three-component serial concatenated IRCC-URC-STC scheme, where the IRregular Convolutional Code (IRCC) [29], [30] and Unity-Rate Code (URC) [31] are employed to facilitate the near-capacity performance on the end-to-end link, as discussed in [32][34], and the Space-Time Code (STC) is used to achieve spatial diversity gains and/or coding gains. On the other hand, at both of the two relays, the same two-component serial concatenated IRCC-STC scheme is employed for the successive relaying aided network considered in this contribution, where the IRCCs at the relays may have different coding rates and weighting coefficients, and will assist us in attaining a near-capacity performance, as in [22]. The notations $\pi_{s}$ and $\pi_{r_{i}}$ seen in Fig. 2 represent the bit-wise random interleavers used at the source $s$ and the relay $r_{i}$, respectively. Since the frames transmitted and relayed from the source and relays have the same frame length, as seen in Fig. 1(d), we choose the same average code rate $R$ for the different IRCCs used at the source and relays of the twin-relay aided network. Note that there is no interference between the two relays during any of their transmission phases, as detailed in [25]. Hence, no interference suppression is needed at the relays, as seen in Fig. 2. However, at the destination, except for the first and last phase, suppression of the interference becomes a potential problem, which will be detailed in the next subsection.

\section{B. Iterative Decoding at the Destination}

In contrast to the iterative decoder of the conventional single-relay-aided network of [22], Fig. 3 illustrates our novel frame-by-frame successive interference cancellation (SIC) aided iterative decoder designed for the Ir-CSTC scheme of the successive relaying aided network. The SIC aided iterative decoder of Fig. 3 has two distinctive parts. Except for the first and last phase of the $(N+1)$-phase relaying protocol, in each intermediate phase, the destination first uses the iterative SIC algorithm [34] to separate the signals received from the source and relays. For example, in Phase $n$ of Fig. 3, the destination receives the signals of frames $(n-1)$ and $n$ from the relay $r_{j}, j=1$ or 2 as well as from the source $s$, respectively. It first detects the signals of frame $(n-1)$ using SIC, while treating the signals of frame $n$ as interference. After frame $(n-1)$ is detected, the destination subtracts it from the received signals and proceeds to detect frame $n$. As discussed in [34], after a sufficient number of SIC iterations near-optimum performance 


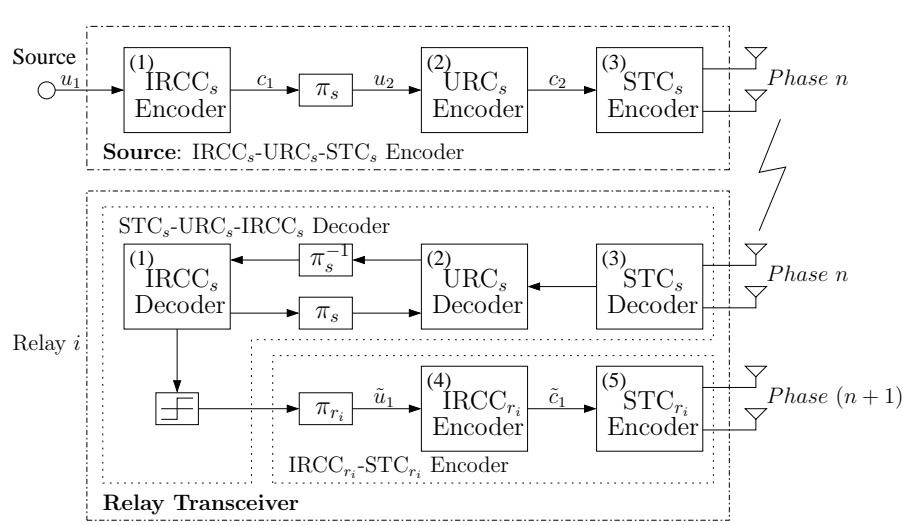

Fig. 2. Schematic of the Ir-CSTC encoder for the successive relaying aided network.

may be achieved for both frames $(n-1)$ and $n$. After the iterative SIC operations, the appropriately separated signals of frame $(n-1)$ received from the relay $r_{j}$ are used in the ensuing iterative decoding process of Fig. 3 in conjunction with the detected signals of frame $(n-1)$ received from the source $s$ in Phase $(n-1)$. The detected signals of frame $n$ are then decoded by the $\mathrm{URC}_{s}$ decoder seen in the middle of the top part of Fig. 3 in order to produce the a priori LogLikelihood Ratio (LLR) values $L_{1, a}\left(c_{1}\right)$ of the coded bits $c_{1}$ by the Maximum Aposteriori Probability (MAP) algorithm [35]. The $\mathrm{IRCC}_{s}$ decoder seen at the top right of Fig. 3 processes the information forwarded by the $\mathrm{URC}_{s}$ decoder in conjunction with the a priori LLR values $L_{1, a}\left(u_{1}\right)$ of the information bits $u_{1}$ gleaned by the " $\mathrm{STC}_{r}-\mathrm{IRCC}_{r}$ " relay-decoder in order to generate the a posteriori LLR values $L_{1, p}\left(u_{1}\right)$ and $L_{1, p}\left(c_{1}\right)$ of the information bits $u_{1}$ and of the coded bits $c_{1}$, respectively. As seen in Fig. 3, the a priori LLRs $L_{1, a}\left(c_{1}\right)$ are subtracted from the a posteriori LLR values $L_{1, p}\left(c_{1}\right)$ and then they are fed back to the $\mathrm{URC}_{s}$ decoder as the a priori information $L_{2, a}\left(u_{2}\right)$ through the interleaver $\pi_{s}$. We term this informationexchange process seen in the top trace of Fig. 3 as the "inner" iteration". Similarly, during the "outer" iterations, the a priori LLR values $L_{1, a}\left(u_{1}\right)$ fed into the $\operatorname{IRCC}_{s}$ decoder are also subtracted from the a posteriori LLR values $L_{1, p}\left(u_{1}\right)$ for the sake of generating the extrinsic LLR values $L_{1, e}\left(u_{1}\right)$, as seen at the top right corner of Fig. 3. Then $L_{1, e}\left(u_{1}\right)$ is passed to the amalgamated " $\mathrm{STC}_{r}-\mathrm{IRCC}_{r}$ " relay-decoder as the a priori information $L_{4, a}\left(\tilde{u}_{1}\right)$ through the interleaver $\pi_{r}$ in conjunction with the signals received from the relay $r_{i}, i=2$ or 1 during Phase $(n+1)$ in order to compute the a posteriori LLR values $L_{4, p}\left(\tilde{u}_{1}\right)$ of the permuted information bits $\tilde{u}_{1}$. As seen in Fig. 3 , the extrinsic information $L_{4, e}\left(\tilde{u}_{1}\right)$ is generated by subtracting the a priori information $L_{4, a}\left(\tilde{u}_{1}\right)$ from the $a$ posteriori information $L_{4, p}\left(\tilde{u}_{1}\right)$, before $L_{4, e}\left(\tilde{u}_{1}\right)$ is fed back to the $\mathrm{IRCC}_{s}$ decoder as the a priori information $L_{1, a}\left(u_{1}\right)$ through the de-interleaver $\pi_{r}^{-1}$. During the last "outer" iteration, the LLR values $L_{1, p}\left(u_{1}\right)$ of the original information bits $u_{1}$ are passed to the hard-decision block of Fig. 3 in order to estimate

\footnotetext{
${ }^{5}$ Explicitly, at the destination node, the extrinsic information exchange between the $\mathrm{URC}_{s}$ decoder and the $\mathrm{IRCC}_{s}$ decoder is referred to as the "inner" iteration, while that between the parallel amalgamated " $\mathrm{STC}_{s}-\mathrm{URC}_{s}-\mathrm{IRCC}_{s}$ " decoder and the amalgamated " $\mathrm{STC}_{r}-\mathrm{IRCC}_{r}$ " relay-decoder is referred to as the "outer" iteration.
}

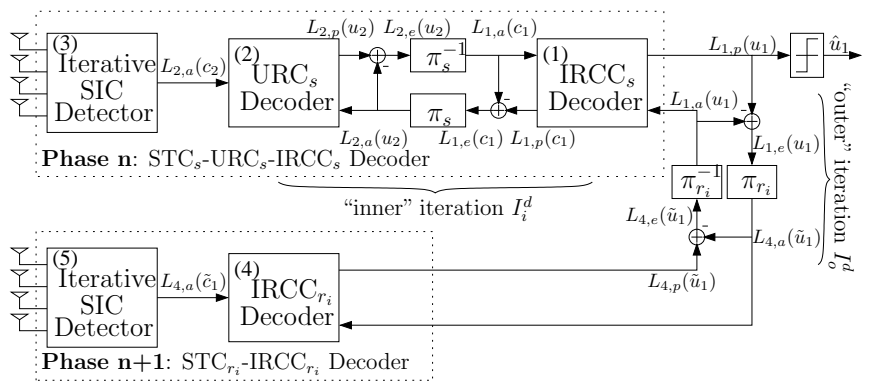

Fig. 3. A frame-by-frame SIC aided iterative decoder of the Ir-CSTC scheme at the destination.

the source bits.

Similarly, in the subsequent Phase $(n+1)$, the signals of frame $n$ received from the relay $r_{i}$ and frame $(n+1)$ received from the source $s$ are detected consecutively by the iterative SIC algorithm at the destination. The detected signals of frame $n$ are passed to the amalgamated " $\mathrm{STC}_{r_{i}}$ IRCC $_{r_{i}}$ " decoder and are used in the ensuing iterative decoding process. The separated signals of frame $(n+1)$ will then be used in the subsequent three-stage iterative decoding process in conjunction with the detected signals of frame $(n+1)$ in Phase $(n+2)$. This process continues, until the last frame is decoded.

\section{NeAR-CAPACITy System Design BASED on EXIT CHARTS}

As presented in [22], the proposed Ir-DSTC coding scheme is capable of near-capacity cooperative communications in the context of a conventional single-relay-aided network. In this section, we will demonstrate that our general Ir-CSTC scheme is also capable of achieving decoding convergence to an infinitesimally low Bit Error Ratio (BER) at Signalto-Noise Ratios (SNRs) close to the DCMC capacity limit (constrained information rate) of the successive relaying channel. We first derive the upper and lower bounds of the CCMC capacity as well as the constrained-information-rate bounds for the successive relaying system, when employing Alamouti's STBC scheme [36], as detailed in Section IV-A. Then, the EXIT chart based joint source-and-relay mode design will be carried out in Section IV-B. In Section IV-C, the EXIT chart analysis of a specific example will be given to demonstrate the proficiency of the code design procedure proposed in Section IV-B.

\section{A. Capacity and Information-Rate Bounds}

As presented in [4], a general upper bound on the CCMC capacity of a half-duplex ${ }^{6}$ relay system is derived based on the original work on the full-duplex relay channels [3], which is given by

$$
\begin{gathered}
C_{\mathrm{CCMC}}^{\mathrm{coop}} \leq \max _{p\left(x_{1}, x_{2}, x\right)}\left\{\operatorname { m i n } \left\{\lambda E\left[I\left(X_{1} ; Y_{1}, Y\right)\right]+(1-\lambda) E\left[I\left(X_{2} ; Y_{2} \mid X\right)\right]\right.\right. \\
\left.\left.\lambda E\left[I\left(X_{1} ; Y_{1}\right)\right]+(1-\lambda) E\left[I\left(X_{2}, X ; Y_{2}\right)\right]\right\}\right\}
\end{gathered}
$$

${ }^{6}$ In [4], the source node continues transmission during the second time slot. 
where $p\left(x_{1}, x_{2}, x\right)$ indicates the joint probability of the signals transmitted from the source and the relay nodes, while $\lambda$ is the ratio of the first time slot duration to the total frame duration. The signals $X_{1}$ and $X_{2}$ are transmitted from the source node during the first and the second time slot, respectively, while $Y_{1}$ and $Y_{2}$ represent the corresponding signals received at the destination during the two consecutive time slots. Furthermore, $Y$ and $X$ are the received and transmitted signals at the relay node, respectively. On the other hand, another achievable rate definition for the DF protocol, which can be regarded as a lower bound on the CCMC capacity of the relay system, was provided in [4] in the form of

$$
\begin{gathered}
C_{\mathrm{CCMC}}^{\mathrm{coop}} \geq \max _{p\left(x_{1}, x_{2}, x\right)}\left\{\operatorname { m i n } \left\{\lambda E\left[I\left(X_{1} ; Y\right)\right]+(1-\lambda) E\left[I\left(X_{2} ; Y_{2} \mid X\right)\right],\right.\right. \\
\left.\left.\lambda E\left[I\left(X_{1} ; Y_{1}\right)\right]+(1-\lambda) E\left[I\left(X_{2}, X ; Y_{2}\right)\right]\right\}\right\},
\end{gathered}
$$

where $I(A ; B)$ represents the mutual information between the channel's input $A$ and the corresponding output $B$, and $E(\cdot)$ denotes the expectation with respect to the fading coefficients.

Referring to the transmission frame structure of Fig. 1(d), the transmission arrangement of our twin-relay-aided successive relaying network can be treated as a superposition of the transmissions of two half-duplex three-terminal networks [4] minus a single direct source-to-destination link, provided that the number of frames is sufficiently high. Hence, we may readily derive the upper and lower bounds on the CCMC capacity of our successive relaying channel as

$$
\begin{aligned}
& C_{\mathrm{CCMC}}^{\mathrm{coop}} \leq \max _{p\left(c_{s}, c_{r_{1}}, c_{r_{2}}\right)}\left\{\operatorname { m i n } \left\{\frac{1}{2} E\left[I\left(C_{s}^{1} ; Y_{d}^{1}, Y_{r_{1}}\right)\right]\right.\right. \\
& \left.+\frac{1}{2} E\left[I\left(C_{s}^{2} ; Y_{d}^{2} \mid C_{r_{1}}\right)\right], \frac{1}{2} E\left[I\left(C_{s}^{1} ; Y_{d}^{1}\right)\right]+\frac{1}{2} E\left[I\left(C_{s}^{2}, C_{r_{1}} ; Y_{d}^{2}\right)\right]\right\} \\
& \quad+\min \left\{\frac{1}{2} E\left[I\left(C_{s}^{1} ; Y_{d}^{1}, Y_{r_{2}}\right)\right]+\frac{1}{2} E\left[I\left(C_{s}^{2} ; Y_{d}^{2} \mid C_{r_{2}}\right)\right]\right. \\
& \left.\left.\frac{1}{2} E\left[I\left(C_{s}^{1} ; Y_{d}^{1}\right)\right]+\frac{1}{2} E\left[I\left(C_{s}^{2}, C_{r_{2}} ; Y_{d}^{2}\right)\right]\right\}-E\left[I\left(C_{s} ; Y_{d}\right)\right]\right\}
\end{aligned}
$$

and

$$
\begin{aligned}
& C_{\mathrm{CCMC}}^{\mathrm{coop}} \geq \max _{p\left(c_{s}, c_{r_{1}}, c_{r_{2}}\right)}\left\{\operatorname { m i n } \left\{\frac{1}{2} E\left[I\left(C_{s}^{1} ; Y_{r_{1}}\right)\right]\right.\right. \\
& \left.+\frac{1}{2} E\left[I\left(C_{s}^{2} ; Y_{d}^{2} \mid C_{r_{1}}\right)\right], \frac{1}{2} E\left[I\left(C_{s}^{1} ; Y_{d}^{1}\right)\right]+\frac{1}{2} E\left[I\left(C_{s}^{2}, C_{r_{1}} ; Y_{d}^{2}\right)\right]\right\} \\
& \quad+\min \left\{\frac{1}{2} E\left[I\left(C_{s}^{1} ; Y_{r_{2}}\right)\right]+\frac{1}{2} E\left[I\left(C_{s}^{2} ; Y_{d}^{2} \mid C_{r_{2}}\right)\right]\right. \\
& \left.\left.\frac{1}{2} E\left[I\left(C_{s}^{1} ; Y_{d}^{1}\right)\right]+\frac{1}{2} E\left[I\left(C_{s}^{2}, C_{r_{2}} ; Y_{d}^{2}\right)\right]\right\}-E\left[I\left(C_{s} ; Y_{d}\right)\right]\right\}
\end{aligned}
$$

respectively, where $C_{s}=\left\{\begin{array}{ll}C_{s}^{1}, & 1^{s t} \text { time slot } \\ C_{s}^{2}, & 2^{\text {nd }} \text { time slot }\end{array}\right.$ and $Y_{d}=$ $\left\{Y_{d}^{1}, \quad 1^{s t}\right.$ time slot

$\begin{cases}Y_{d}^{2}, & 2^{\text {nd }} \text { time slot }\end{cases}$ pendent of each other in most practical scenarios, (7) and (8) can be simplified to

$$
\begin{aligned}
& C_{\mathrm{CCMC}}^{\mathrm{coop}} \leq \max _{p\left(c_{s}, c_{r_{1}}, c_{r_{2}}\right)}\left\{\min \left\{\frac{1}{2} E\left[I\left(C_{s}^{1} ; Y_{d}^{1}, Y_{r_{1}}\right)\right], \frac{1}{2} E\left[I\left(C_{s}^{2}, C_{r_{1}} ; Y_{d}^{2}\right)\right]\right\}\right. \\
&\left.+\min \left\{\frac{1}{2} E\left[I\left(C_{s}^{1} ; Y_{d}^{1}, Y_{r_{2}}\right)\right], \frac{1}{2} E\left[I\left(C_{s}^{2}, C_{r_{2}} ; Y_{d}^{2}\right)\right]\right\}\right\}
\end{aligned}
$$

and

$$
\begin{aligned}
C_{\mathrm{CCMC}}^{\mathrm{coop}} & \geq \max _{p\left(c_{s}, c_{r_{1}}, c_{r_{2}}\right)}\left\{\min \left\{\frac{1}{2} E\left[I\left(C_{s}^{1} ; Y_{r_{1}}\right)\right], \frac{1}{2} E\left[I\left(C_{s}^{2}, C_{r_{1}} ; Y_{d}^{2}\right)\right]\right\}\right. \\
+ & \left.\min \left\{\frac{1}{2} E\left[I\left(C_{s}^{1} ; Y_{r_{2}}\right)\right], \frac{1}{2} E\left[I\left(C_{s}^{2}, C_{r_{2}} ; Y_{d}^{2}\right)\right]\right\}\right\},
\end{aligned}
$$

respectively.

In addition to the CCMC capacity bounds, we also evaluate the information-rate bounds for the successive relaying channel in conjunction with i.u.d discrete inputs. Thus, the upper and lower constrained information-rate bounds are given by

$$
\begin{aligned}
C_{\mathrm{DCMC}}^{\mathrm{coop}} & \leq \min \left\{\frac{1}{2} E\left[I_{d}\left(C_{s}^{1} ; Y_{d}^{1}, Y_{r_{1}}\right)\right], \frac{1}{2} E\left[I_{d}\left(C_{s}^{2}, C_{r_{1}} ; Y_{d}^{2}\right)\right]\right\} \\
+\min & \left\{\frac{1}{2} E\left[I_{d}\left(C_{s}^{1} ; Y_{d}^{1}, Y_{r_{2}}\right)\right], \frac{1}{2} E\left[I_{d}\left(C_{s}^{2}, C_{r_{2}} ; Y_{d}^{2}\right)\right]\right\}
\end{aligned}
$$

and

$$
\begin{aligned}
C_{\mathrm{DCMC}}^{\mathrm{coop}} & \geq \min \left\{\frac{1}{2} E\left[I_{d}\left(C_{s}^{1} ; Y_{r_{1}}\right)\right], \frac{1}{2} E\left[I_{d}\left(C_{s}^{2}, C_{r_{1}} ; Y_{d}^{2}\right)\right]\right\} \\
+ & \min \left\{\frac{1}{2} E\left[I_{d}\left(C_{s}^{1} ; Y_{r_{2}}\right)\right], \frac{1}{2} E\left[I_{d}\left(C_{s}^{2}, C_{r_{2}} ; Y_{d}^{2}\right)\right]\right\},
\end{aligned}
$$

respectively, where the subscript $d$ indicates the i.u.d discreteinput constraint.

In this contribution, we employ Alamouti's 4QAM-based G2 scheme at both the source and relay nodes, where the network model is configured with $N_{s}=2, N_{r_{1}}=N_{r_{2}}=2$ and $N_{d}=4$, as shown in Figs. 2 and 3. The codeword matrix of the G2 scheme is given by:

$$
\mathbf{C}_{G 2}=\left(\begin{array}{cc}
c_{1} & c_{2} \\
-\bar{c}_{2} & \bar{c}_{1}
\end{array}\right)^{T} .
$$

Based on Eqs. (1) and (4), the signal received at the relay node $r_{i}$ during $V=2$ consecutive symbol periods can be written as:

$$
\mathbf{Y}_{r_{i}}=\sqrt{G_{s r_{i}}} \mathbf{H}_{s r_{i}} \mathbf{C}_{s}+\mathbf{N}_{r_{i}}
$$

and except for the first and last phase, the signal received at the destination node during $V=2$ consecutive symbol periods in the intermediate phase can be written as:

$$
\mathbf{Y}_{d}=\sqrt{G_{s d}} \mathbf{H}_{s d} \mathbf{C}_{s}+\sqrt{G_{r_{i} d}} \mathbf{H}_{r_{i} d} \mathbf{C}_{r_{i}}+\mathbf{N}_{d}
$$

respectively, where $\mathbf{Y}_{r_{i}}=\left[\mathbf{y}_{r_{i}, 1}, \ldots, \mathbf{y}_{r_{i}, V}\right] \in \mathbb{C}^{N_{r_{i}} \times V}$ and $\mathbf{Y}_{d}=$ $\left[\mathbf{y}_{d, 1}, \ldots, \mathbf{y}_{d, V}\right] \in \mathbb{C}^{N_{d} \times V}$ are the matrices hosting the sampled signal received at the relay node $r_{i}$ and the destination node, respectively. Furthermore, $\mathbf{C}_{s}=\left[\mathbf{c}_{s, 1}, \ldots, \mathbf{c}_{s, V}\right] \in \mathbb{C}^{N_{s} \times V}$ and $\mathbf{C}_{r_{i}}=\left[\mathbf{c}_{r_{i}, 1}, \ldots, \mathbf{c}_{r_{i}, V}\right] \in \mathbb{C}^{N_{r_{i}} \times V}$ represent Alamouti's G2 matrices characterizing the transmissions of the source and relay $r_{i}$, while $\mathbf{N}_{r_{i}}=\left[\mathbf{n}_{r_{i}, 1}, \ldots, \mathbf{n}_{r_{i}, V}\right] \in \mathbb{C}^{N_{r_{i}} \times V}$ and $\mathbf{N}_{d}=$ $\left[\mathbf{n}_{d, 1}, \ldots, \mathbf{n}_{d, V}\right] \in \mathbb{C}^{N_{d} \times V}$ represents the AWGN matrix incurred at relay $r_{i}$ and the destination, respectively. 
Hence, the G2 codeword-matrix-input constrained information-rate bounds of (11) and (12) are given, respectively, by

$$
\begin{aligned}
& C_{\mathrm{DCMC}}^{\mathrm{coop}-\mathrm{g} 2} \leq \min \left\{\frac{1}{2} E\left[I_{d}\left(\mathbf{C}_{s}^{1} ; \mathbf{Y}_{d}^{1}, \mathbf{Y}_{r_{1}}\right)\right], \frac{1}{2} E\left[I_{d}\left(\mathbf{C}_{s}^{2}, \mathbf{C}_{r_{1}} ; \mathbf{Y}_{d}^{2}\right)\right]\right\} \\
& +\min \left\{\frac{1}{2} E\left[I_{d}\left(\mathbf{C}_{s}^{1} ; \mathbf{Y}_{d}^{1}, \mathbf{Y}_{r_{2}}\right)\right], \frac{1}{2} E\left[I_{d}\left(\mathbf{C}_{s}^{2}, \mathbf{C}_{r_{2}} ; \mathbf{Y}_{d}^{2}\right)\right]\right\}
\end{aligned}
$$

and

$$
\begin{aligned}
C_{\mathrm{DCMC}}^{\mathrm{coop}-\mathrm{g} 2} & \geq \min \left\{\frac{1}{2} E\left[I_{d}\left(\mathbf{C}_{s}^{1} ; \mathbf{Y}_{r_{1}}\right)\right], \frac{1}{2} E\left[I_{d}\left(\mathbf{C}_{s}^{2}, \mathbf{C}_{r_{1}} ; \mathbf{Y}_{d}^{2}\right)\right]\right\} \\
+\min & \left\{\frac{1}{2} E\left[I_{d}\left(\mathbf{C}_{s}^{1} ; \mathbf{Y}_{r_{2}}\right)\right], \frac{1}{2} E\left[I_{d}\left(\mathbf{C}_{s}^{2}, \mathbf{C}_{r_{2}} ; \mathbf{Y}_{d}^{2}\right)\right]\right\} .
\end{aligned}
$$

Considering a relay channel associated with perfect sourceto-relay links, i.e. $G_{s r}=\infty$, where the relay nodes are very close to the source node and are capable of perfectly recovering all the information transmitted from the source node, we have

$$
\max _{p\left(c_{s}, c_{r}\right)} E\left[I\left(C_{s} ; Y_{d}, Y_{r}\right)\right] \rightarrow \infty, \quad \max _{p\left(c_{s}, c_{r}\right)} E\left[I\left(C_{s} ; Y_{r}\right)\right] \rightarrow \infty,
$$

and

$$
E\left[I_{d}\left(\mathbf{C}_{s} ; \mathbf{Y}_{d}, \mathbf{Y}_{r}\right)\right]=2, \quad E\left[I_{d}\left(\mathbf{C}_{s} ; \mathbf{Y}_{r}\right)\right]=2,
$$

since the 4QAM-based G2 STC is used. Therefore, the CCMC capacity upper and lower bounds of (9) and (10) converge to

$$
\begin{aligned}
C_{\mathrm{CCMC}}^{\mathrm{coop}}=\max _{p\left(c_{s}, c_{r_{1}}, c_{r_{2}}\right)}\left\{\frac{1}{2} E\left[I\left(C_{s}^{2}, C_{r_{1}} ; Y_{d}^{2}\right)\right]\right. \\
\left.+\frac{1}{2} E\left[I\left(C_{s}^{2}, C_{r_{2}} ; Y_{d}^{2}\right)\right]\right\} .
\end{aligned}
$$

Similarly, the lower and upper bounds on the information rates constrained by the i.u.d G2 codeword-matrix inputs converge to

$$
\begin{aligned}
C_{\mathrm{DCMC}}^{\mathrm{coop}-\mathrm{g} 2}=\min \left\{\frac{1}{2} E\right. & {\left.\left[I_{d}\left(\mathbf{C}_{s}^{2}, \mathbf{C}_{r_{1}} ; \mathbf{Y}_{d}^{2}\right)\right], 1\right\} } \\
& +\min \left\{\frac{1}{2} E\left[I_{d}\left(\mathbf{C}_{s}^{2}, \mathbf{C}_{r_{2}} ; \mathbf{Y}_{d}^{2}\right)\right], 1\right\} .
\end{aligned}
$$

Finally, we reformulate (21) as follows

$$
\begin{array}{r}
C_{\mathrm{DCMC}}^{\mathrm{coop}-\mathrm{g} 2}\left(\mathrm{SNR}_{e}^{\text {coop }}\right)=\min \left\{\frac{1}{2} C_{\mathrm{DCMC}^{\mathrm{s}} 1->\mathrm{d}}^{\mathrm{S}}\left(\mathrm{SNR}_{e}^{s}, \mathrm{SNR}_{e}^{r_{1}}, G_{r_{1} d}\right), 1\right\} \\
+\min \left\{\frac{1}{2} C_{\mathrm{DCMC}^{\mathrm{sr}}->\mathrm{d}}^{\mathrm{rr}_{2}}\left(\mathrm{SNR}_{e}^{s}, \mathrm{SNR}_{e}^{r_{2}}, G_{r_{2} d}\right), 1\right\}
\end{array}
$$

where we refer to $\mathrm{SNR}_{e}^{s}, \mathrm{SNR}_{e}^{r_{1}}$ and $\mathrm{SNR}_{e}^{r_{2}}$ as the equivalent $\mathrm{SNRs}^{7}$ at the source and relays, respectively, which are all equal to $P_{0} / N_{0}$, since they transmit at the same power of $P_{0}$. Furthermore, $\mathrm{SNR}_{e}^{\text {coop }}$ is the equivalent overall SNR of the successive relaying aided network, which is defined by $\mathrm{SNR}_{e}^{\text {coop }}=P_{0} / N_{0}$. The variables $C^{\mathrm{sr}_{1}->\mathrm{d}}$ and $C^{\mathrm{sr}_{2}->\mathrm{d}}$ represent the corresponding multiple-access channels' capacities, which can be similarly computed with the aid of Monte-Carlo techniques based on Eq. (11) of [34].

\footnotetext{
${ }^{7}$ Here we introduced the terminology of equivalent $\mathrm{SNR}_{e}$ to define the ratio of the signal power at the transmitter side with respect to the noise level at the receiver side, as in [26]. Although this does not have a direct physical interpretation, it simplifies our discussions.
}

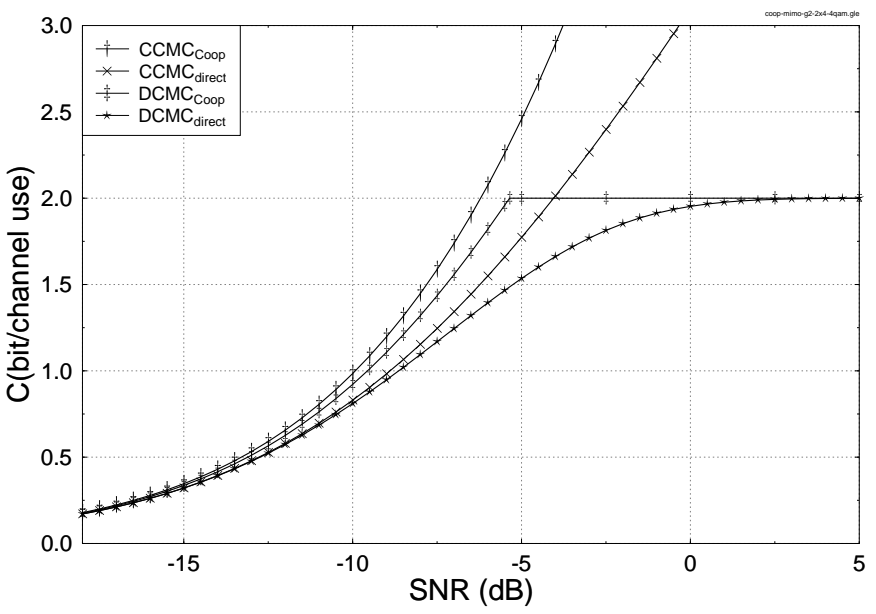

Fig. 4. The CCMC capacity curve and constrained information rates employing Alamouti's G2 scheme with $G_{s r_{1}}=G_{s r_{2}}=\infty$ and $G_{r_{1} d}=G_{r_{2} d}=1$ for the successive relaying channel.

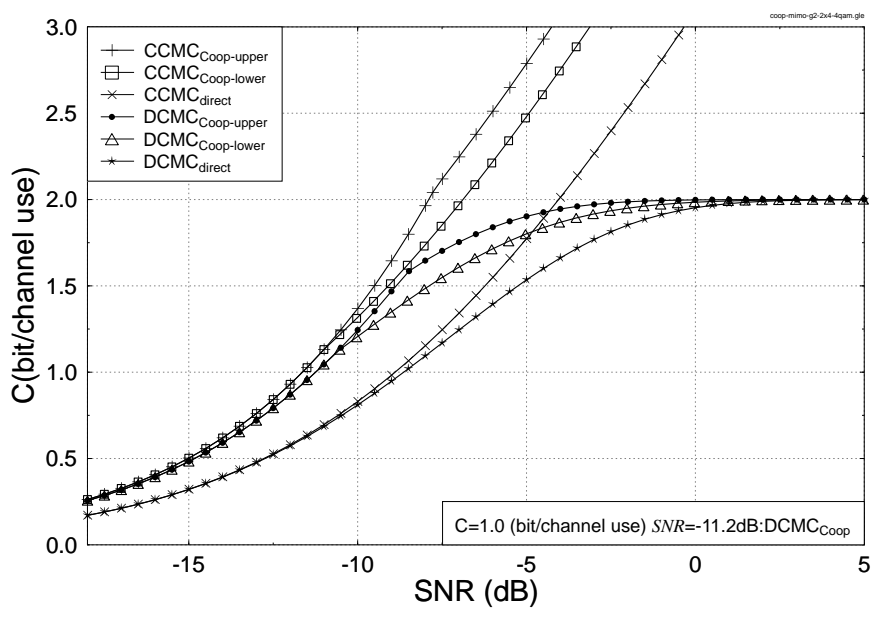

Fig. 5. The CCMC capacity curve and constrained information rates employing Alamouti's G2 scheme with $G_{s r_{1}}=G_{s r_{2}}=8$ and $G_{r_{1} d}=G_{r_{2} d}=2$ for the successive relaying channel.

An example is given in Fig. 4 for the successive relaying aided uncorrelated Rayleigh fading channel with $G_{r_{1} d}=$ $G_{r_{2} d}=1$. Based on Eqs. (20), (21) and (22), we characterize the CCMC capacity and the information rates obeying the i.u.d 4QAM-based G2 codeword-matrix-input constraint. The capacity and information rates of the direct source-to-destination link channel are also depicted in Fig. 4, where we assume that the power constraint is $2 P_{0}$ for the direct transmission based benchmarker for a fair comparison. We can observe in Fig. 4 that the information-rate gain is substantial and the factor 0.5 multiplexing loss imposed by creating a separate transmit and receive slot is recovered compared to the classic direct transmission.

Furthermore, we consider a more practical example for a specific network associated with $G_{s r_{1}}=G_{s r_{2}}=8$ and $G_{r_{1} d}=$ $G_{r_{2} d}=2$ in Fig. 5, where the source-to-relay links are imperfect. The CCMC capacity and the information rates are evaluated by the upper and lower bounds given in (9)-(10) and (11)-(12). In this scenario, we can see in Fig. 5 that the lower and upper bounds converge in the low and medium SNR 
regimes below a certain convergence threshold. Next, we will show in Section IV-C that the optimized cooperative coding scheme is capable of performing near-perfectly at the relay nodes, despite having imperfect source-to-relay links. The substantial capacity gains shown in Fig. 5 may be achieved by using successive relaying techniques instead of classic direct transmissions, partially, because the factor 0.5 multiplexing loss of [22] is recovered.

\section{B. Joint Code Design at the Source and Relay Nodes}

For the sake of near-capacity cooperative communications in the successive relaying aided network in this section, we generalize the joint source-and-relay mode design procedure of [22] to suit our four-terminal network of Fig. 1, which is summarised as follows:

Step 1: Choose a specific average code rate $R$ for the $\mathrm{IRCC}_{s}$ at the source and employ the EXIT curve matching algorithm of [29] at the relays in order to obtain the optimized weighting coefficients $\alpha_{i}, i=1, \ldots, 17$ of $\mathrm{IRCC}_{s}$, where a narrow but marginally open EXIT-tunnel is created between the EXIT curves of the inner amalgamated " $\mathrm{STC}_{s}-\mathrm{URC}_{s}$ " decoder of Fig. 3 and the outer $\mathrm{IRCC}_{s}$ decoder at the relays. This implies that a near-capacity performance may be achieved for the SR links. Then we store the value of the corresponding transmit power required at the source.

Step 2: Choose the same transmit power at the source as stored in Step 1. Fix the optimized $\mathrm{IRCC}_{s}$ weighting coefficients $\alpha_{i}, i=1, \ldots, 17$ obtained in Step 1 at the source. Then perform iterative decoding by exchanging extrinsic information between the amalgamated " $\mathrm{STC}_{s}-\mathrm{URC}_{s}$ " decoder of Fig. 3 and the $\mathrm{IRCC}_{S}$ decoder at the destination, until the further increase of the area $A_{E}$ under the EXIT curve of the amalgamated " $\mathrm{STC}_{s}-\mathrm{URC}_{s}-\mathrm{IRCC}_{s}$ " decoder of Fig. 3 becomes marginal. Then stop this "inner" iterative decoding process.

Step 3: Assume perfectly error-free DF relaying and the same transmit power at the relay $r_{1}$ as that of the source in the second EXIT chart, which examines the evolution of the input/output mutual information exchanges in the three-stage iterative decoder of the Ir-CSTC scheme. Use the EXIT curve matching algorithm of [29] to match the SNR-dependent EXIT curve of the amalgamated " $\mathrm{STC}_{r_{1}}-\mathrm{IRCC}_{r_{1}}$ " decoder of Fig. 3 to the target EXIT curve of the amalgamated " $\mathrm{STC}_{s}-\mathrm{URC}_{s}$ $\mathrm{IRCC}_{s}$ " decoder observed in Step 2. If an open EXIT-tunnel fails to appear, increase the transmit power at both the source $s$ and the relay $r_{1}$, until a narrow-but-open EXIT-tunnel emerges. Obtain the optimized weighting coefficients $\beta_{j}, j=1, \ldots, 17$ of IRCC $_{r_{1}}$.

Step 4: Repeat the operations outlined in Step 3 for relay $r_{2}$. Step 5: Finally, choose the higher of the two transmit power values obtained in Step 3 and Step 4 as the ultimate transmit power at the source and relays.

\section{EXIT Charts Analysis}

In this contribution, we consider the same average code rate $R=0.5$ for the IRCCs at the source and relays, and the specific successive relaying aided network geometry associated with $G_{s r_{1}}=G_{s r_{2}}=8$ and $G_{r_{1} d}=G_{r_{2} d}=2$. Hence, the effective

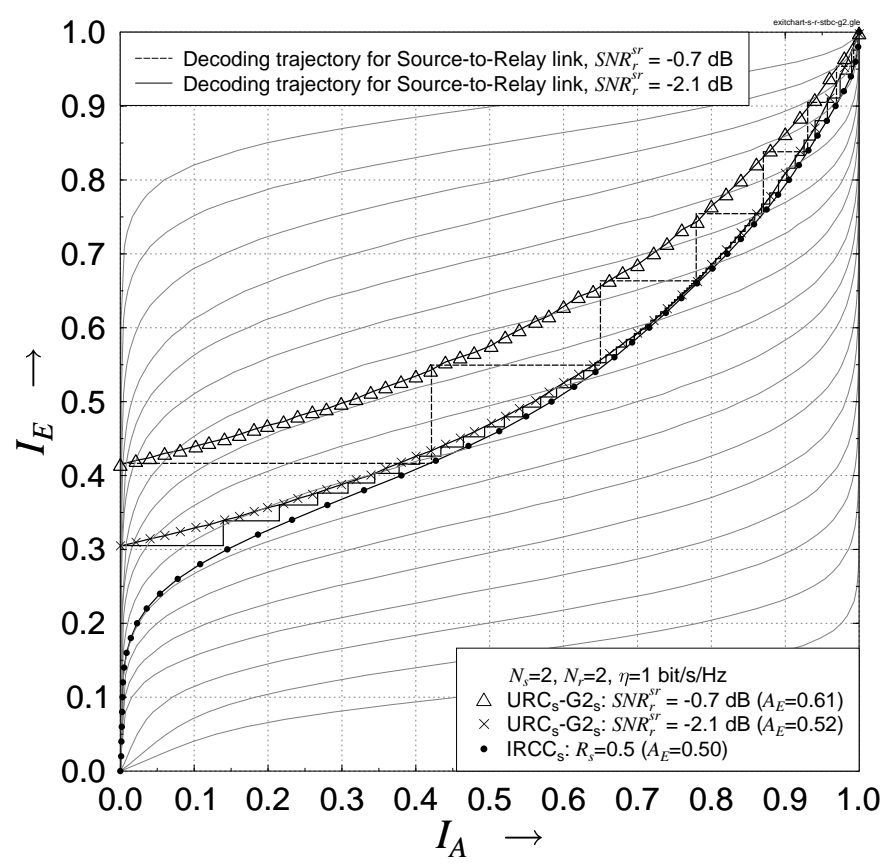

Fig. 6. The EXIT chart curves of the $\mathrm{URC}_{s}-\mathrm{G} 2_{s}$, the $\mathrm{IRCC}_{s}$ with optimized weighting coefficients $\left[\alpha_{1}, \ldots, \alpha_{17}\right]=[0,0,0,0,0,0.327442,0.186505$, $0.113412,0,0.0885527,0,0.0781214,0.0962527,0.0114205,0.0346015$, $0.0136955,0.0500168$ ] and 17 IRCC subcodes for the $(2 \times 2)$ SR link where the $\mathrm{SNR}_{r}^{s r}$ is the receive SNR at the relay.

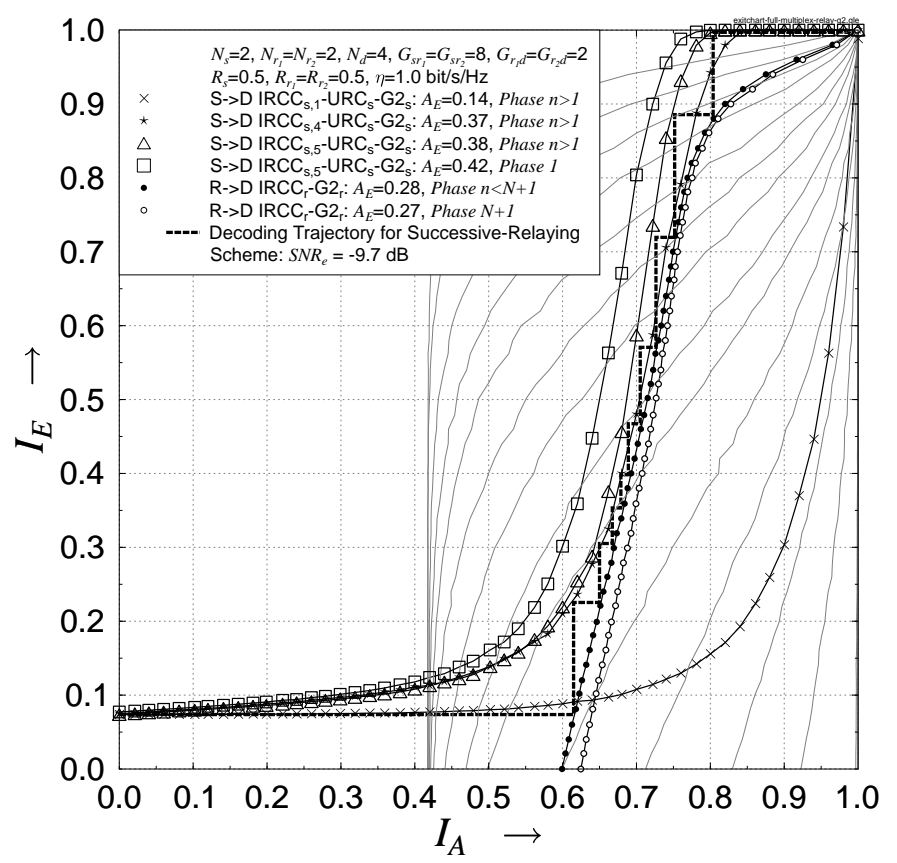

Fig. 7. The EXIT chart curves for the $\mathrm{IRCC}_{s}-\mathrm{URC}_{s}-\mathrm{G} 2_{s}$ with various "inner" iterations, the $\mathrm{IRCC}_{r}-\mathrm{G} 2_{r}$ with $\mathrm{IRCC}_{r}$ having optimized weighting coefficients $\left[\beta_{1}, \ldots, \beta_{17}\right]=[0,0,0,0,0.233115,0.0158742,0.292084,0.220065$, $0.0151108,0,0,0,0,0,0,0,0.22375]$ and 17 SNR-dependent $\mathrm{IRCC}_{r}-\mathrm{G} 2_{r}$ subcodes. The subscript of IRCC $_{s}$ denotes the number of "inner" iterations between the $\mathrm{IRCC}_{s}$ and "G2 $2_{s}-\mathrm{URC}_{s}$ " decoders and the $\mathrm{SNR}_{e}$ represents the equivalent $\mathrm{SNR}$ at the source and relays. 
network throughput is $\frac{N}{N+1} R \log _{2} 4 \approx 1.0 \mathrm{bit} / \mathrm{s} / \mathrm{Hz}$, when 4QAM is employed and the number of frames $N$ is sufficiently high. Since the network is geographically symmetrical, we will not differentiate the relays $r_{1}$ and $r_{2}$ for simplicity. Fig. 6 depicts the EXIT chart of the serial concatenated IRCC $_{S}$ $\mathrm{URC}_{s}-\mathrm{STBC}_{s}$ scheme of the SR link, where $I_{A}$ and $I_{E}$ indicate the mutual informations between the information bits and the a priori LLR values as well as the corresponding extrinsic LLR values, respectively. The EXIT curve of the outer IRCC $_{s}$ having optimized weighting coefficients $\alpha_{i}$ was constructed using the curve matching algorithm of [29] as shown in Fig. 6 and the Monte-Carlo-simulation based decoding trajectories are computed for a frame length of 250000 bits. As seen from Fig. 6, a narrow but marginally open EXIT tunnel emerges for the $(2 \times 2)$ SR communication link. A receive SNR of about $-2.1 \mathrm{~dB}$ is needed in order to attain a decoding convergence to an infinitesimally low BER. As a benefit of the geometricalgain of the SR communication link, the equivalent SNR at the source can be expressed as:

$$
\mathrm{SNR}_{e}^{s}=\mathrm{SNR}_{r}^{s r}-10 \log _{10}\left(G_{s r}\right)[\mathrm{dB}] .
$$

Hence, the minimum $\mathrm{SNR}_{e}^{s}$ at the source required for the sake of obtaining vanishingly low BERs at the relay is -11.1 $\mathrm{dB}$. Since we assume that the source and relays transmit at the same power, we have $\mathrm{SNR}_{e}^{\text {coop }}=\mathrm{SNR}_{e}^{s}=\mathrm{SNR}_{e}^{r}$. Following the design procedure of Section IV-B, a 'wider-than-necessary' EXIT tunnel ${ }^{8}$ is created in the EXIT chart of Fig. 6 at the receive $\mathrm{SNR}$ of $-0.7 \mathrm{~dB}$ at the relay, which corresponds to an equivalent SNR of $-9.7 \mathrm{~dB}$ based on Eq. (23) at the source and relays. Accordingly, it is clearly seen in the EXIT chart of Fig. 7 at the destination that after 5 "inner" iterations between the $\mathrm{IRCC}_{s}$ decoder and the amalgamated " $\mathrm{STBC}_{s}-\mathrm{URC}_{s}$ " decoder, the increase of the area $A_{E}$ under the amalgamated " $\mathrm{STBC}_{s}-\mathrm{URC}_{s}-\mathrm{IRCC}_{s}$ " decoder's EXIT curve becomes marginal. The resultant matching EXIT curve of the amalgamated "STBC ${ }_{r}$ IRCC $_{r}$ " decoder is shown in Fig. 7, where the IRCC $r$ has the optimized weighting coefficients $\beta_{j}$, as summarized in Fig. 7. Similarly, as discussed in [22], the 'narrow-but-open' EXIT-tunnel of Fig. 7 indicates the possibility of achieving decoding convergence to an infinitesimally low BER at near-capacity SNRs for the Ir-CSTC scheme in the successive relaying aided network. This prediction is verified in Fig. 7 by plotting the corresponding Monte-Carlo simulation-based decoding trajectory, which indeed reaches the $(1.0,1.0)$ point of the EXIT chart. Furthermore, we also plot the EXIT curves of the amalgamated " $\mathrm{STBC}_{s}-\mathrm{URC}_{s}$ $\mathrm{IRCC}_{s}$ " decoder in Phase 1 and the amalgamated "STBC ${ }_{r}$ IRCC $_{r}$ " decoder in Phase $(N+1)$ in Fig. 7, respectively. Since the tunnels in Phase 1 and Phase $(N+1)$ are wider, the trajectories can traverse through the tunnels at a reduced number of iterations to reach the $(1.0,1.0)$ point.

\section{Simulation Results and Discussions}

In this section, we characterize the BER versus equivalent overall SNR performance of both the perfect and imperfect

\footnotetext{
${ }^{8} \mathrm{~A}$ 'wider-than-necessary' EXIT tunnel indicates the possibility of reaching the convergence $(1.0,1.0)$ point for fewer iterations, which involves lower complexity.
}

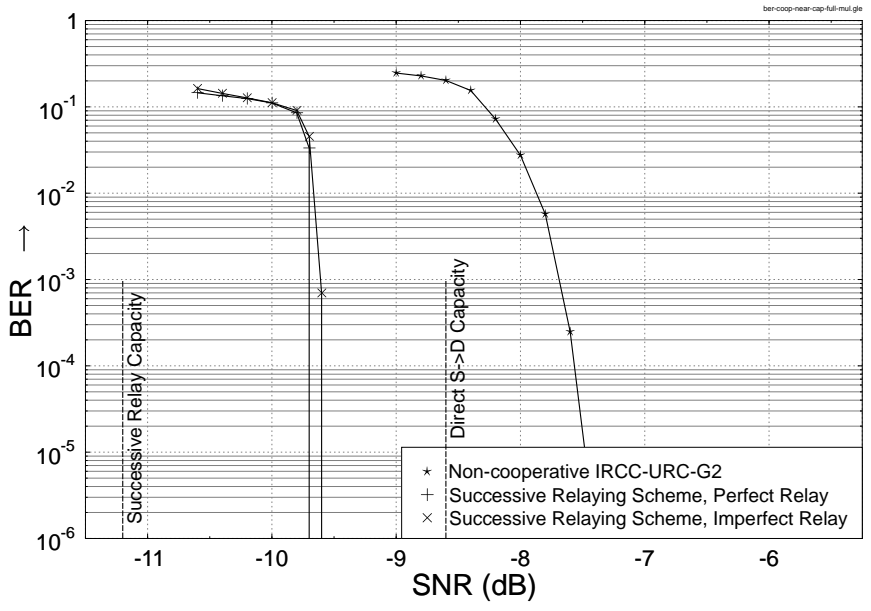

Fig. 8. BER versus equivalent SNR performance of both perfect and imperfect relaying aided Ir-CSTC schemes in the successive relaying aided network for a frame length of 250000 bits, while the performance of the non-cooperative system is also depicted here for comparison.

relaying aided Ir-CSTC schemes in the successive relaying aided network as well as that of a non-cooperative IRCCURC-STBC scheme in Fig. 8. According to the trajectory predictions seen in Figs. 6 and 7, for the cooperative space-time coding scheme, the number of decoding iterations between the $\mathrm{IRCC}_{s}$ decoder and the amalgamated "STBC ${ }_{s}-\mathrm{URC}_{S}$ " decoder was fixed to $I^{r}=11$ at the relays. At the destination, the number of "inner" decoding iterations was fixed to $I_{i}^{d}=5$, while the number of "outer" decoding iterations between the parallel amalgamated " $\mathrm{STBC}_{s}-\mathrm{URC}_{s}-\mathrm{IRCC}_{s}$ " decoder and the amalgamated "STBC $r$-IRCC $r$ " decoder was fixed to $I_{o}^{d}=11$. On the other hand, for the non-cooperative serial concatenated IRCC-URC-STBC scheme, we employ an outer IRCC, which has the same weighting coefficients $\alpha_{i}$ as that of the $\mathrm{IRCC}_{s}$ in the cooperative system. The number of decoding iterations exchanging extrinsic information between the outer IRCC decoder and the inner "STBC-URC" decoder was fixed to $I_{n o n}=11$ as well. It is clearly seen in Fig. 8 that the performance of the perfect relaying-aided scheme matches the EXIT chart predictions of Fig. 7, while the imperfect relaying-aided scheme performs similarly to the perfect relaying scheme. This is due to the fact that the source information is detected without decoding errors after a sufficiently high number of decoding iterations at the relays. On the other hand, the Ir-CSTC scheme employed in the successive relaying aided network outperforms the non-cooperative serial concatenated IRCC-URC-STBC scheme by about $2.2 \mathrm{~dB}$, while maintaining the same effective throughput. As portrayed in Fig. 8, the IrCSTC scheme is capable of performing within $1.5 \mathrm{~dB}$ of the corresponding successive relaying channel's DCMC capacity.

\section{CONCLUSIONS}

In this contribution, we have proposed a general irregular cooperative space-time coding scheme for the successive relaying aided network. The CCMC capacity and the constrained information-rate bounds of Alamouti's STBC scheme were derived for the successive relaying aided channel. It was observed in Figs. 4 and 5 that the factor 0.5 multiplexing 
loss of the single-relay-aided network may be recovered by the successive relaying protocol with the aid of an additional relay, which is more practical and feasible than the fullduplex system. The generalized joint source-and-relay mode design procedure advocated is capable of finding the optimal cooperative coding scheme, which performs close to the capacity limit. Furthermore, the code design procedure is not limited to a specific networking scenario, it is applicable under virtually any network configuration. Moreover, it is generically applicable, regardless of the specific choice of the space-time codes at the source and relays as well as of the irregular components, as exemplified by the family of irregular low-density parity-check (LDPC) codes, etc. as long as the inherent irregularity at the source and relays holds. Finally, our numerical results seen in Fig. 8 demonstrated that the joint source-and-relay mode design based on EXIT chart analysis is capable of near-capacity cooperative communications in the twin-relay successive relaying aided network. A more practical scenario, where the interference between the relays is taken into account, will be considered in our future work. In that scenario interference suppression is needed not only at the destination but also at the relays.

\section{REFERENCES}

[1] A. Sendonaris, E. Erkip, and B. Aazhang, "User cooperation diversity: Part I and II," IEEE Transactions on Communications, vol. 51, pp. 19271948, Nov. 2003.

[2] J. Laneman, D. Tse, and G. Wornell, "Cooperative diversity in wireless networks: Efficient protocols and outage behavior," IEEE Transactions on Information Theory, vol. 50, pp. 3062-3080, Dec. 2004.

[3] T. M. Cover and A. E. Gamal, "Capacity theorems for the relay channel," IEEE Transactions on Information Theory, vol. 25, pp. 572-584, Sept. 1979.

[4] A. Høst-Madsen and J. Zhang, "Capacity bounds and power allocation for wireless relay channel," IEEE Transactions on Information Theory, vol. 51, pp. 2020-2040, Jun. 2005.

[5] R. U. Nabar, H. Bölcskei, and F. W. Kneubühler, "Fading relay channels: Performance limits and space-time signal design," IEEE Journal on Selected Areas on Communications, vol. 22, pp. 1099-1109, Aug. 2004

[6] G. Kramer, M. Gastpar, and P. Gupta, "Cooperative strategies and capacity theorems for relay networks," IEEE Transactions on Information Theory, vol. 51, pp. 3037-3063, Sept. 2005.

[7] B. Wang, J. Zhang, and A. Host-Madsen, "On the capacity of mimo relay channels," IEEE Transactions on Information Theory, vol. 51, pp. 2943, Jan. 2005.

[8] A. Reznik, S. R. Kulkarni, and S. Verdu, "Capacity and optimal resource allocation in the degraded Gaussian relay channel with multiple relays," in 40th Allerton Conference on Communication, Control, and Computing, pp. 377-386, Oct. 2002.

[9] B. Rankov and A. Wittneben, "Achievable Rate Regions for the Twoway Relay Channel," in IEEE International Symposium on Information Theory, (Seattle, USA), pp. 1668-1672, July 2006.

[10] K. Azarian, H. El Gamal, and P. Schniter, "On the achievable diversitymultiplexing tradeoff in half-duplex cooperative channels," IEEE Transactions on Information Theory, vol. 51, pp. 4152-4172, Dec. 2005.

[11] T. Hunter and A. Nosratinia, "Cooperation diversity through coding," in IEEE International Symposium on Information Theory, pp. 220-, 2002.

[12] B. Wang and J. Zhang, "MIMO relay channel and its applications for cooperative communication in ad hoc networks," in 41st Allerton Conference on Communication, Control, and Computing, pp. 15561565, Oct. 2003.

[13] J. Laneman and G. Wornell, "Distributed space-time-coded protocols for exploiting cooperative diversity in wireless networks," IEEE Transactions on Information Theory, vol. 49, pp. 2415-2425, Oct. 2003.

[14] Y. Fan, C. Wang, J. Thompson, and H. Poor, "Recovering multiplexing loss through successive relaying using repetition coding," IEEE Transactions on Wireless Communications, vol. 6, pp. 4484-4493, December 2007.
[15] B. Zhao and M. Valenti, "Distributed turbo coded diversity for relay channel," Electronics Letters, vol. 39, pp. 786-787, May 2003.

[16] S. X. Ng, Y. Li and L. Hanzo, "Distributed turbo trellis coded modulation for cooperative communications," in ICC'09, (Dresden, Germany), 1418 June 2009.

[17] S. ten Brink, "Convergence behaviour of iteratively decoded parallel concatenated codes," IEEE Transactions on Communications, vol. 49, pp. 1727-1737, Oct. 2001.

[18] S. ten Brink, "Designing iterative decoding schemes with the extrinsic information transfer chart," AEU International Journal of Electronics and Communications, vol. 54, pp. 389-398, Nov. 2000.

[19] Z. Zhang and T. Duman, "Capacity-approaching turbo coding and iterative decoding for relay channels," IEEE Transactions on Communications, vol. 53, pp. 1895-1905, Nov. 2005.

[20] Z. Zhang and T. Duman, "Capacity-approaching turbo coding for half-duplex relaying," IEEE Transactions on Communications, vol. 55 pp. 1895-1906, Oct. 2007.

[21] A. Conti, V. Tralli, and M. Chiani, "Pragmatic space-time codes for cooperative relaying in block fading channels," EURASIP Journal on Advances in Signal Processing, vol. 2008.

[22] L. Kong, S. X. Ng, R. G. Maunder and L. Hanzo, "Irregular Distributed Space-Time Code Design for Near-Capacity Cooperative Communications," in IEEE VTC'09 (Fall), (Anchorage, Alaska, USA), 20-23 September 2009.

[23] J. G. Proakis, Digital Communications. 4th ed. New York: McGraw-Hill, 2001.

[24] S. X. Ng and L. Hanzo, "On the MIMO channel capacity of multidimensional signal sets," IEEE Transactions on Vehicular Technology, vol. 55, pp. 528-536, March 2006.

[25] S. Yang and J.-C. Belfiore, "On slotted amplify-and-forward cooperative diversity schemes," 2006 IEEE International Symposium on Information Theory, pp. 2446-2450, July 2006.

[26] H. Ochiai, P. Mitran, and V. Tarokh, "Design and analysis of collaborative diversity protocols for wireless sensor networks," IEEE 60th Vehicular Technology Conference, 2004 Fall, vol. 7, pp. 4645-4649 Vol. 7, Sept. 2004.

[27] J. Luo, R. S. Blum, L. J. Cimini, L. J. Greenstein, and A. M. Haimovich, "Decode-and-forward cooperative diversity with power allocation in wireless networks," IEEE Transactions on Wireless Communications, vol. 6, pp. 793-799, March 2007.

[28] L. Zuari, A. Conti and V. Tralli, "Effects of relay position and power allocation in space-time coded cooperative wireless systems," in the sixth IEEE International Symposium on Wireless Communication Systems, (Siena-Tuscany, University of Siena, Italy), pp. 700-704, 7-10 Sept. 2009.

[29] M. Tüchler and J. Hagenauer, "EXIT charts of irregular codes," in Proceeding of the 36th Annual Conference on Information Sciences and Systems [CDROM], (Princeton, NJ, USA), March 2002.

[30] M. Tüchler, "Design of serially concatenated systems depending on the block length," IEEE Transactions on Communications, vol. 52, pp. 209218, Feb. 2004.

[31] D. Divsalar, S. Dolinar and F. Pollara, "Serial turbo trellis coded modulation with rate-1 inner code," in ISIT, (Sorrento, Italy), p. 194 25-30 June 2000.

[32] S. X. Ng and S. Das and J. Wang and L. Hanzo, "Near-Capacity Iteratively Decoded Space-Time Block Coding," in IEEE VTC'08 (Spring), (Marina Bay, Singapore), pp. 590-594, 11-14 May 2008.

[33] L. Kong, S. X. Ng and L. Hanzo, "Near-capacity three-stage downlink iteratively decoded generalized layered space-time coding with low complexity," in GLOBECOM'08, (New Orleans, LA, USA), pp. 1-6, 30 Nov.-04 Dec. 2008.

[34] L. Kong, S. X. Ng, R. Y. S. Tee, R. G. Maunder, and L. Hanzo, "Reduced-complexity near-capacity downlink iteratively decoded generalized multi-layer space-time coding using irregular convolutional codes," IEEE Transactions on Wireless Communications, vol. 9, pp. 684695, Feb. 2010.

[35] L. Hanzo, T. H. Liew and B. L. Yeap, Turbo Coding, Turbo Equalisation and Space Time Coding for Transmission over Wireless channels. New York, USA: John Wiley IEEE Press, 2002.

[36] S. M. Alamouti, "A simple transmit diversity technique for wireless communications," IEEE Journal on Selected Areas in Communications, vol. 16, pp. 1451-1458, Oct. 1998 


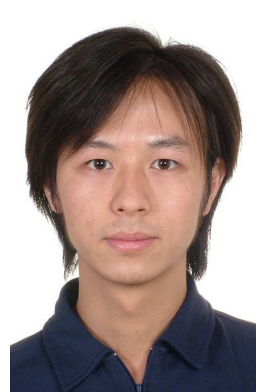

Lingkun Kong received the B.Eng. degree in information engineering and the M.Eng. degree in communications and signal processing from Southeast University, Nanjing, China, in 2004 and 2006, respectively. $\mathrm{He}$ is currently working towards the Ph.D. degree with the Communications Research Group, School of Electronics and Computer Science, University of Southampton, UK. His research interests include channel coding, space-time coding, iterative detection techniques as well as co-located and distributed MIMO systems.

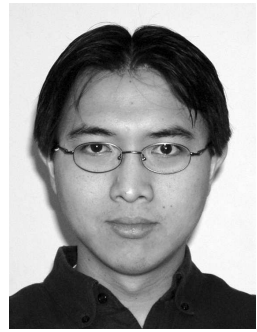

Soon Xin Ng (S'99-M'03-SM'08) received the B.Eng. degree (First class) in electronics engineering and the Ph.D. degree in wireless communications from the University of Southampton, Southampton, U.K., in 1999 and 2002, respectively. From 2003 to 2006, he was a postdoctoral research fellow working on collaborative European research projects known as SCOUT, NEWCOM and PHOENIX. Since August 2006, he has been a lecturer in wireless communications at the University of Southampton. He has been part of a team working on the OPTIMIX European project since March 2008. His research interests include adaptive coded modulation, channel coding, space-time coding, joint source and channel coding, OFDM, MIMO, cooperative communications and distributed coding. He has published numerous papers and coauthored a book in this field.

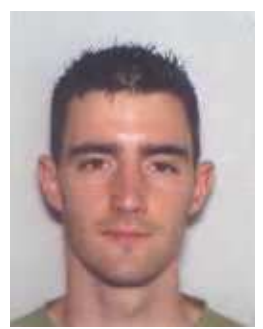

Robert G. Maunder has studied with the School of Electronics and Computer Science, University of Southampton, UK, since October 2000. He was awarded a first class honours BEng in Electronic Engineering in July 2003, as well as a PhD in Communications and a lectureship in December 2007. Rob's research interests include joint source/channel coding, iterative decoding and irregular coding. He has published a number of IEEE papers in these areas.

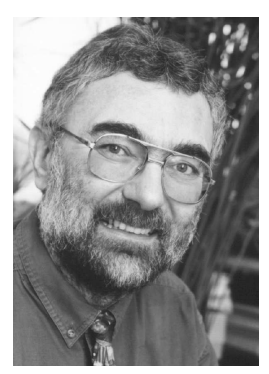

Lajos Hanzo (M'91-SM'92-F'04) FREng, FIEEE, FIET, DSc received his degree in electronics in 1976 and his doctorate in 1983. During his 31-year career in telecommunications he has held various research and academic posts in Hungary, Germany and the UK. Since 1986 he has been with the School of Electronics and Computer Science, University of Southampton, UK, where he holds the chair in telecommunications. He has co-authored 17 books on mobile radio communications totalling in excess of 10000 pages, published in excess of 800 research papers, acted as TPC Chair of IEEE conferences, presented keynote lectures and been awarded a number of distinctions. Currently he is directing an academic research team, working on a range of research projects in the field of wireless multimedia communications sponsored by industry, the Engineering and Physical Sciences Research Council (EPSRC) UK, the European IST Programme and the Mobile Virtual Centre of Excellence (VCE), UK. $\mathrm{He}$ is an enthusiastic supporter of industrial and academic liaison and he offers a range of industrial courses. He is also an IEEE Distinguished Lecturer as well as a Governor of both the IEEE ComSoc and the VTS. He is the acting Editor-in-Chief of the IEEE Press. For further information on research in progress and associated publications please refer to http://www-mobile.ecs.soton.ac.uk. 